\title{
Different knockout genotypes of OsIAA23 in rice using CRISPR/Cas9 generating different phenotypes
}

\author{
Mengmeng Jiang ${ }^{1} \cdot$ Huaying $\mathrm{Hu}^{1} \cdot$ Jing Kai ${ }^{1} \cdot$ Milton Brian Traw ${ }^{1} \cdot$ Sihai Yang ${ }^{1} \cdot$ Xiaohui Zhang $^{1}$
}

Received: 23 October 2018 / Accepted: 11 April 2019 / Published online: 19 April 2019

(c) The Author(s) 2019

\begin{abstract}
Key message We have isolated several Osiaa23 rice mutants with different knockout genotypes, resulting in different phenotypes, which suggested that different genetic backgrounds or mutation types influence gene function.

Abstract The Auxin/Indole-3-Acetic Acid (Aux/IAA) gene family performs critical roles in auxin signal transduction in plants. In rice, the gene OsIAA23 (Os06t0597000) is known to affect development of roots and shoots, but previous knockouts in OsIAA23 have been sterile and difficult for research continuously. Here, we isolate new Osiaa23 mutants using the CRISPR/Cas9 system in japonica (Wuyunjing24) and indica (Kasalath) rice, with extensive genome re-sequencing to confirm the absence of off-target effects. In Kasalath, mutants with a 13-amino acid deletion showed profoundly greater dwarfing, lateral root developmental disorder, and fertility deficiency, relative to mutants with a single amino acid deletion, demonstrating that those 13 amino acids in Kasalath are essential to gene function. In Wuyunjing24, we predicted that mutants with a single base-pair frameshift insertion would experience premature termination and strong phenotypic defects, but instead these lines exhibited negligible phenotypic difference and normal fertility. Through RNA-seq, we show here that new mosaic transcripts of OSIAA23 were produced de novo, which circumvented the premature termination and thereby preserved the wild-type phenotype. This finding is a notable demonstration in plants that mutants can mask loss of function CRISPR/Cas9 editing of the target gene through de novo changes in alternative splicing.
\end{abstract}

Keywords Oryza sativa $\cdot$ OsIAA23 $\cdot$ CRISPR/Cas9 $\cdot$ Root development $\cdot$ Alternative splicing

\section{Introduction}

Auxins were the first major phytohormones discovered and orchestrate cardinal plant developmental processes, such as cell elongation, cell division and differentiation, tropic responses to light and gravity, general root and shoot

Mengmeng Jiang and Huaying Hu contributed equally to this work.

Electronic supplementary material The online version of this article (https://doi.org/10.1007/s11103-019-00871-5) contains supplementary material, which is available to authorized users.

Sihai Yang

sihaiyang@nju.edu.cn

Xiaohui Zhang

xiaohuizhang@nju.edu.cn

1 State Key Laboratory of Pharmaceutical Biotechnology, School of Life Sciences, Nanjing University, Nanjing 210023, China architecture, organ patterning, vascular development, growth in tissue culture, and apical dominance (Woodward and Bartel 2005; Teale et al. 2006). Auxin signal transduction is controlled by interaction between auxin/indole-3-acetic acid (Aux/IAA) and auxin response factor (ARF) proteins (Liscum and Reed 2002). When cellular auxin levels are low, Aux/IAA proteins are stable and form heterodimers with ARF proteins, thereby repressing expression of the target auxin response genes. Elevated cellular auxin levels activate the auxin receptor, transport inhibitor response 1/auxin signaling F-box protein (TIR1/AFB), leading to ubiquitylation and degradation of Aux/IAA proteins through the SCFTIR1/ AFBs complex, thereby releasing ARF proteins to activate auxin response genes (Luo et al. 2015).

The Aux/IAA gene family members encode short-lived nuclear proteins characterized by four highly conserved domains, designated as domains I, II, III, and IV (Mukesh et al. 2006). Domain I harbors an amino-terminal leucine repeat motif (LxLxLx) and functions as a transcriptional repressor of downstream auxin-regulated genes (Tiwari et al. 
2004). Domain II contains a conserved TIR1/AFB recognition sequence, GWPPV, and plays a major role in the stability of Aux/IAA proteins. Mutations in the GWPPV recognition sequence block rapid degradation of Aux/IAAs, thus disturbing the auxin signaling pathway (Worley et al. 2000; $\mathrm{Ni}$ et al. 2011). ARF proteins also harbor Domains III and IV, which are involved in homo- and heterodimerization of Aux/IAA and/or ARF proteins (Ouellet et al. 2001).

The plant Aux/IAA family is also large and diverse, consisting of 29 and 31 members in Arabidopsis thaliana and rice, respectively (Liscum and Reed 2002; Mukesh et al. 2006). The Aux/IAA gene family has also been identified in other plant species such as Populus, maize, potato, grape, soybean, and Brassica (Çakir et al. 2012; Ludwig et al. 2013; Singh and Jain 2015; Gao et al. 2016; Li et al. 2017). In Arabidopsis, several gain-of-function dominant or semi-dominant mutants of Aux/IAA genes have been characterized and found to be related to curl patterns in leaves, light and gravitropic responses, and plant length, particularly lateral root formation (Reed 2001; Overvoorde et al. 2005). Most of these lines have amino acid mutations occur within the core sequence of Domain II. For example, the Arabidopsis mutants iaa3 (Tian et al. 2002), iaa7 (Mai et al. 2011), iaal7 (Ouellet et al. 2001), iaa19 (Tatematsu et al. 2004), iaa28 (Rogg et al. 2001), and iaal4 (Fukaki et al. $2002,2005)$ show varying degrees of lateral root developmental defects. On the other hand, single T-DNA insertion mutants in 12 of the $29 A u x / I A A$ family members and double or triple mutants of closely related $A u x / I A A$ genes show no visible developmental defects compared to the wild-type, suggesting extensive functional redundancy among members of the Aux/IAA gene family (Overvoorde et al. 2005). In rice, overexpression and mutant analysis are the major methods of Aux/IAA gene function research. Overexpression of OSIAA4 leads to dwarfism, increased tiller angles, and reduced gravity response (Song and Xu 2013). OsIAA6 is found to be induced by drought stress and related to tiller outgrowth (Jung et al. 2015). The mutants Osiaa3, Osiaal1, and Osiaal3 also show lateral root developmental defects, similar to Arabidopsis mutants (Nakamura et al. 2006; Zhu et al. 2012; Kitomi et al. 2012; Zhang et al. 2018). Ni et al. found that the OSIAA23 gene plays an important role in postembryonic maintenance of the quiescent center in rice by a semi-dominant mutant (Ni et al. 2011, 2014). This mutant harbored an amino acid mutation within the core sequence of Domain II, which causes "GWPPV" to become "EWPPV".

Except for the genes mentioned above, the function of most $A u x / I A A$ genes in rice has not been elucidated. Because of functional redundancy of the $A u x / I A A$ genes, the traditional methods (e.g. T-DNA insertions, knockdowns and point mutations) have yielded few mutants with visible defective phenotypes. Currently, non-synonymous mutations of the core sequence of Domain II are the most effective way to study the Aux/IAA genes. However, it is generally difficult to generate mutants with expected mutations via traditional artificial mutagenesis. The clustered regulatory interspaced short palindromic repeat/CRISPRassociated protein 9 (CRISPR/Cas9) gene editing technology is an effective approach that solves this problem (Miao et al. 2013). In addition, mutations within the core sequence GWPPV of Domain II often cause significant developmental defects. Indeed, Osiaa23 homologous mutants show extremely low levels of fertility, making it inconvenient to preserve mutants and thus hindering subsequent studies on OsIAA23 (Ni et al. 2011). Kepinski and Leyser (2004) reported that a short sequence of 17 amino acids (AKAQVVGWPPVRNYRKN) around the core sequence of Domain II is sufficient for auxin-regulated interaction. Therefore, we aimed to obtain mutants with reduced developmental defects in rice by site-directed mutagenesis of amino acids around the core sequence GWPPV using the CRISPR/Cas9 system.

In this research, we analyzed $I A A$ homologs in the rice genome and selected OsIAA23 (Os06t0597000) as the editing target. We adopted a standard method for the CRISPR/ Cas9 system tailored to rice (Miao et al. 2013), and applied it to two rice varieties, Kasalath (indica) and Wuyunjing24 (japonica). To identify off-target effects and confirm accuracy of the targeted changes, we re-sequenced the mutant lines and screened for mutations in a genome-wide scale, with the result that no other effective mutations were found. Similar to the Osiaa23 mutant reported by Ni et al. (2011), the Kasalath mutants with a 13 amino-acid deletion downstream of the core sequence of Domain II also showed dwarfing, lateral root developmental disorders, and fertility deficiencies. However, the Wuyunjing24 mutants with a single base-pair insertion downstream of the core sequence of Domain II showed a weak mutant phenotype, yet exhibited normal fertility. Analysis of RNA sequencing data revealed new transcript isoforms in the Wuyunjing24 mutant, which are predicted to produce functional OsIAA23 proteins. The alternative splicing mechanism thus apparently works in the Wuyunjing24 mutant, resulting in the synthesis of a functional IAA protein. Further expression analysis suggests that the recovered OSIAA23 interacts with its two homologs, OSIAAI and OSIAA15, especially OSIAA15, in a same pathway, thereby preserving the wild-type phenotypes.

\section{Materials and methods}

\section{Sequence analysis of the OsIAA23 homologs in the rice genome}

The IAA genes used in this study were as identified by Mukesh et al. (2006). The CDSs of the IAA genes were first translated into amino acid sequences and aligned using 
MUSCLE as implemented in MEGA7 (Kumar et al. 2016). The alignments were used to construct a neighbor-joining tree with a Kimura 2-parameter model. The nonsynonymous $(K a)$ and synonymous $(K s)$ nucleotide substitutions were calculated based on the Nei-Gojobori method. Nucleotide diversity $(\pi)$ was estimated with Jukes and Cantor correction. Tajima's D was calculated with a default parameter. The genetic parameters were calculated using a Perl script. The rice genome sequences were supplied by Yuan et al. (2017).

\section{Rice materials and growth conditions}

Two rice cultivars, namely, Kasalath (Oryza sativa L. ssp. indica $\mathrm{cv}$. Kasalath) and Wuyunjing24 (O. sativa L. ssp. japonica $\mathrm{cv}$. Wuyunjing24) were selected as the recipient materials. All rice plants, including wild-type and various mutants, were grown in parallel in nutrient-rich soil outdoors within a temperature range of $20-30{ }^{\circ} \mathrm{C}$.

\section{PAM sequence design and vector construction}

The vectors pOs-sgRNA and pH-Ubi-cas9-7 were obtained from Professor Lijia Qu (State Key Laboratory for Protein and Plant Gene Research, Peking-Tsinghua Center for Life Science, College of Life Sciences, Peking University, Beijing, China). The sgRNA guide sequence was designed for targeting OsIAA23 using CRISPR-P (http://crispr.hzau.edu. $\mathrm{cn} / \mathrm{CRISPR} 2 /)$. Default parameters were used to design the guide sequences. Then, the target sequences were checked in the scaffold sequences of Kasalath and Wuyunjing24. An sgRNA situated within Domain II of the OsIAA23 gene was selected. To clone the spacer, two oligos were synthesized and the $5^{\prime}$ ends of the forward and reverse strands were respectively linked to 'ggca' and 'aaac' sequences. The primer sequences containing spacers are listed in Supplementary Table S1. After annealing the oligos, the spacer with overhanging sequences was inserted into the $B s a$ I site of the pOs-sgRNA vector. A Gateway Cloning LR reaction (Invitrogen) of the resulting pOs-sgRNA construct was performed with the $\mathrm{pH}$-Ubi-cas9-7 vector to generate the OsIAA23-targeting sgRNA:Cas9. After the spacer sequence was verified by Sanger sequencing, the construct was transfected into Agrobacterium tumefaciens EHA105 cells.

\section{Stable transformation and mutant screening}

Following a standard approach (Zhang et al. 2015), we transformed the sgRNA:Cas9 vector into two rice varieties (Kasalath and Wuyunjing24) via Agrobacterium-mediated transformation. For each recipient rice variety, at least eight flasks, each containing 10 shell-less seeds were set up for callus tissue culture. During Agrobacterium infection, callus tissues from one or two flasks were transferred to a single plate. A total of 10 plates of callus tissues were prepared for inoculation with Agrobacterium, and at least two transformants from each plate were selected for transplantation. Therefore, at least 20 transformants were developed from different callus lines. Here, independent transgenic events were isolated in the presence of hygromycin B.

For each transformed plant, DNA was extracted from the leaves using the cetyl trimethylammonium bromide (CTAB) method, and the target gene was amplified by PCR. The primer sequences are listed in the Supplementary Table S1. Finally, the PCR products were sequenced to determine whether gene editing was successful. Only the transformed plants of which the target gene was successfully edited were used in the subsequent experiments.

\section{Re-sequencing and mutation screening of mutants}

For each mutant and wide-type plants, DNA was extracted using the CTAB method. All plant DNA were sequenced on Illumina Hiseq 4000 platform with a coverage depth of $\sim 20 \times$ and qualified bases (base quality $\geq 20$ ) over $90 \%$ after removing adaptors and low quality reads (i.e. reads contain half of low-quality bases). Cleaned reads were mapped to the reference genome $O$. sativa L. ssp. japonica var. Nipponbare (IRGSP-1.0) by BWA-mem (version 0.7.10-r789) with default settings (Li 2013). The resulting bam files were then sorted and processed with MarkDuplicates in Picard package (version 1.114) to remove non-informative PCR duplicates. A local realignment step was also implemented using RealignerTargetCreator and IndelRealigner in GATK package (version 3.5.0.22) to reduce false variant calls due to higher alignment errors around INDELs (insertion/deletion) (DePristo et al. 2011). Variant sites, including SNPs (single nucleotide polymorphisms) and INDELs, were called by Sametools and filtered using custom Python scripts. To check putative offtarget effects of CRISPR/Cas9, all the genes of IAA family and ARF family were manually inspected in Intergrative Genomics Viewer (IGV) (Thorvaldsdóttir et al. 2013) to screen out any sequence changes from the wild-type plant. An effective mutation which might influence the phenotype of the mutants was defined when a nucleotide was different from the wild-type sequence(s) and homozygous (the mutated reads $>3$, mapping quality $\geq 50$ and effective matched degree $\geq 90$ ). Here, "effective matched degree" means the ratio of matched length to whole read length. 


\section{Expression analysis of OsIAA23 in mutant and wild-type rice}

Total RNA was prepared from $50 \mathrm{mg}$ tissues from 2-weekold plants or plants in the ripe stage using standard protocols (Takara Code No. 9767). The cDNA samples prepared from no more than $10 \mu \mathrm{g}$ of total RNA in a total amount of $40 \mu \mathrm{l}$ was used as template and mixed with a PrimeScript Master Mix and primers for real-time PCR (RT-PCR) analysis (Takara Code No. RR036A), according to the manufacturer's instructions. Reactions were run in triplicate. The housekeeping gene glyceraldehyde-3-phosphate dehydrogenase $(G 3 P D H)$ was used as an internal control to normalize expression levels. The expression data were analyzed using the $2^{(-\Delta \Delta \mathrm{Ct})}$ method. Each pair of primers designed by Oligo 6 was checked by BLAST in the rice genome sequence to ensure that the amplified sequence was indeed unique. The primer sequences are listed in Supplementary Table S1.

Transcripts from the root tissues of different mutants and wild-type cultivars were PCR amplified using the cDNA library. The PCR DNA fragments were extracted from the agarose gel using a Thermo Scientific GeneJET gel extraction kit. The purified DNA fragments were sequenced in two different ways: by Illumina HiSeq 4000 at a coverage of $200,000 \times$ and by Pacbio Sequel at a coverage of 20,000 $\times$. The database from Illumina HiSeq 4000, combined 56 published data (Supplementary Table S2), was analyzed using Tophat and Samtools. The database of Pacbio Sequel was mapped in the genome by Gmap and cufflinked by Sametools. The exon-intron junctions and transcripts of different mutants were detected and compared using Python.

\section{Results}

\section{Evolutionary features of the OsIAA23 gene in the rice genome}

Thirty-one IAA homologs (IAA1-IAA3I) were identified in the rice genome (Mukesh et al. 2006). To estimate the evolutionary features of these $I A A$ genes, we calculated nucleotide diversity $(\pi), K a / K s$ ratios and Tajima's D of their CDSs and the main four domains (I-IV) among 393 rice genomes. These rice genomes included 166 indica, 167 japonica, and 60 wild rice varieties. As Tajima's D values showed, an excess of low-frequency nucleotide polymorphisms was detected in most IAA genes, particularly in Domain II and IV (Supplementary Table S3). The CDS regions of several $I A A$ genes showed relatively high polymorphism $(\geq 2 \%$ ) such as OsIAA2, OsIAA23, and OSIAA27 (Fig. 1). For some genes, including OsIAA2, OsIAA22, OSIAA23, and OsIAA29, strong selection was detected in their Domain II, which plays a major role in the stability of Aux/IAA proteins.

Phylogenetic reconstruction of the OsIAAs (Fig. 1) shows that $O$ SIAA23 belongs to a small clade, which includes three homologs (OSIAA1, OSIAA15, and

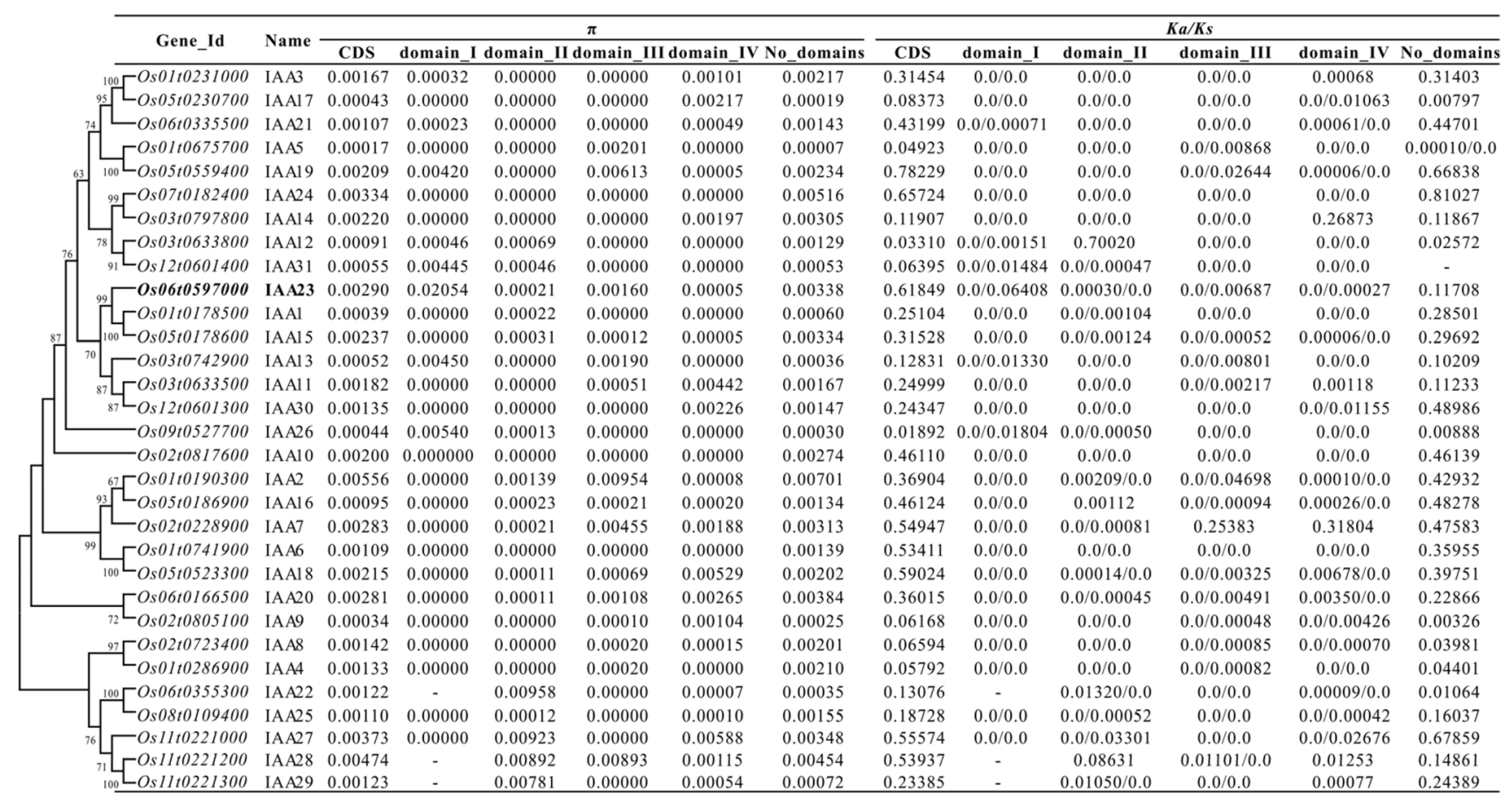

Fig. 1 Nucleotide diversity $(\pi)$ and ratios of nonsynonymous to synonymous nucleotide substitutions $(K a / K s)$ of $I A A$ genes among rice genomes, including 166 indica rice, 167 japonica rice and 60 wild rice 
OSIAA23). The nucleotide diversity between OSIAA23 and OSIAA1, OSIAA15 and OSIAA23, and OSIAA 1 and OsIAA15 is $0.293,0.305$, and 0.158 , respectively. Compared to its two paralogs, OsIAA23 exhibits significantly higher nucleotide diversity whether in CDS region, Domain I, or Domain III. Each gene of the clade is old, with each having alleles in other grasses, such as maize, sorghum, and Brachypodium (data not shown), suggesting the conservation and importance of the three genes in the Poaceae.

At the gene expression level, the OsIAA23 gene and its two paralogs show different tissue expression patterns (Mukesh et al. 2006). OsIAA23 is highly expressed in the roots, whereas OSIAAI and OSIAA15 are highly expressed in flowers. In addition, unlike OSIAAI and OSIAA15, the expression of $O$ SIAA23 is not induced by the auxin analog 2,4-dichlorophenoxyacetic acid (2,4-D). These results suggest that OSIAA23 may have undergone functional differentiation.

\section{Targeted mutagenesis of OsIAA23 using the CRISPR/ Cas9 system}

According to the gene structure and the conserved domains, the specific spacer sequence for OsIAA23 was designed and is located at the end of Domain II (Fig. 2a, b). Two Kasalath mutants and five Wuyunjing24 mutants were identified by sequencing the target region of OSIAA23 in the positive transgenic T0 plants (Table 1). Both heterozygous double knockout mutants were found in the two recipient rice varieties (Table 2). Compared to the wild-type Kasalath, all the T0 Kasalath mutants were slightly dwarf, have less tillers, and semi-sterile. However, all the T0 Wuyunjing24 mutants showed no distinguishable mutant phenotype, as indicated by normal tiller number, seed setting rate, and growth cycle, except for slight dwarfing.

Two mutation types were identified in the Kasalath mutants, namely, a 3-bp deletion that we designated as KaOsiaa23-3, and a 39-bp deletion named KaOsiaa23-39 (Fig. 2b). Similarly, for the Wuyunjing24 mutants, two

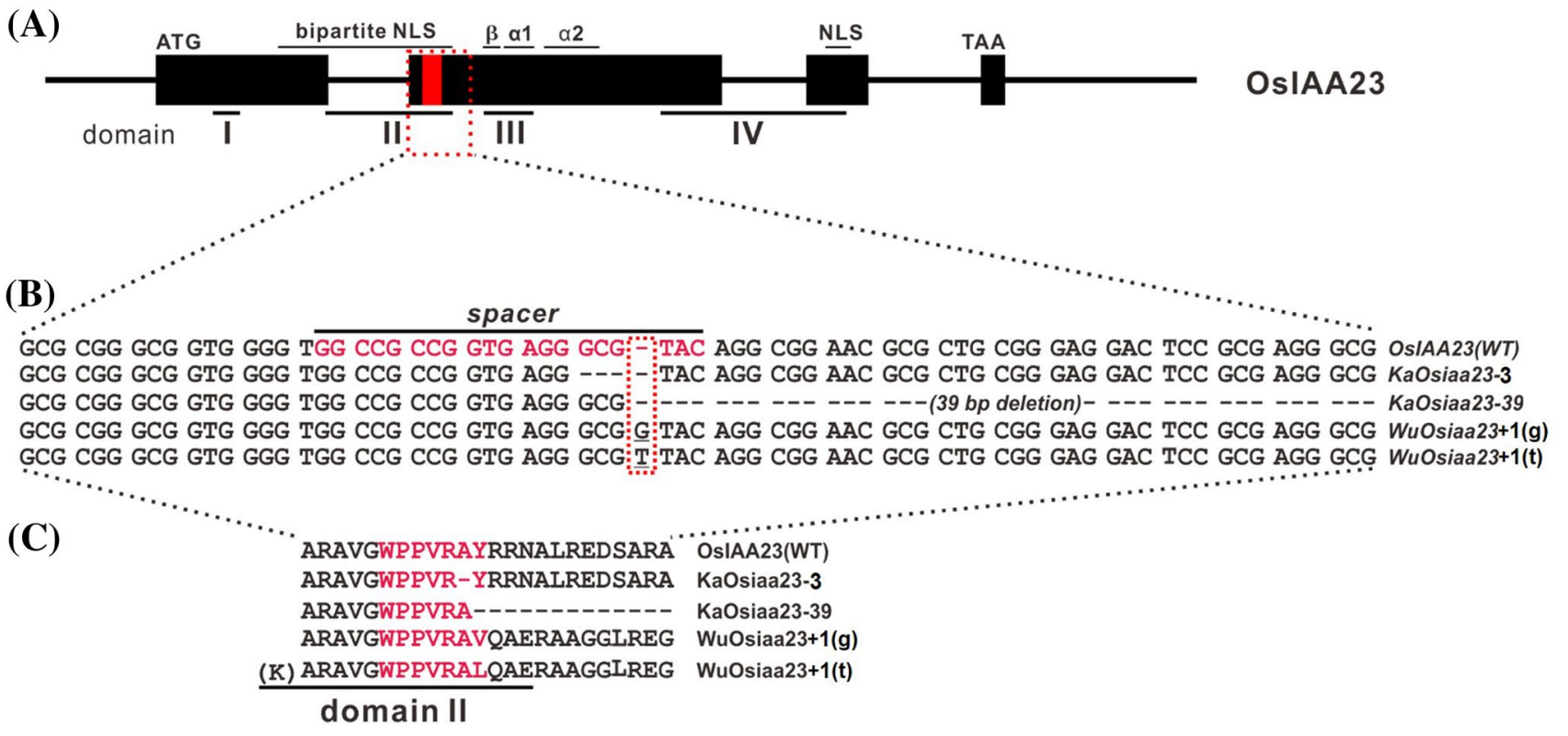

Fig. 2 Targeted mutagenesis of OsIAA23 in rice using CRISPR/ Cas system. a Diagram of gene structure and conserved domains of OsIAA23 (Os06t0597000-01 for representation). The black rectangles represent the exon regions. The short lines are marked with Roman numerals bellow indicate four domains of OsIAA23, and the spacer location is marked by red. b sgRNA:Cas9-induced rice OsIAA23 gene mutations at the target site in the mutants. The sequence marked in the red text under the black line indicates the spacer sequence. c Amino acid sequence alignment of wild type OsIAA23 protein and the Osiaa 23 mutants. The sequence marked in the red text indicated the spacer location, and Domain II is indicated by underlined text
Table 1 CRISPR/Cas9 editing results of OsIAA23 in T0 rice plants

\begin{tabular}{llllll}
\hline Rice varieties & $\begin{array}{l}\text { PCR iden- } \\
\text { tification }\end{array}$ & Mutants & Editing rate (\%) & Editing type & Mutant genotype \\
\hline Kasalath & 6 & 2 & 33.3 & double mutant & 3 bp-deletion/39 bp-deletion \\
Wuyunjing24 & 5 & 5 & 100 & double mutant & G-insertion/T-insertion \\
\hline
\end{tabular}


Table 2 Genotypes and mutant sequences of T0 plants

\begin{tabular}{|c|c|c|c|}
\hline T0 lines & Genotype & Sequence & $\begin{array}{l}\text { Germina- } \\
\text { tion rate } \\
(\%)\end{array}$ \\
\hline KaOsiaa23-1/3/4/5 & wild type & GGGCGTACAGGCGGAACGCGCTGCGGGAGGACGCCGCGAGGGCGAAG & 100 \\
\hline $\mathrm{KaOsiaa23-2/6}$ & 3 bp-deletion/39 bp-deletion & $\begin{array}{l}\text { GG-TACAGGCGGAACGCGCTGCGGGAGGACGCCGCGAGGGCGAAG } \\
\text { GG-GCGAAG }\end{array}$ & 66.7 \\
\hline WuOsiaa23-1/2/3/4/5 & G-insertion/T-insertion & \multicolumn{2}{|c|}{$\begin{array}{l}\text { GGGCGGTACAGGCGGAACGCGCTGCGGGAGGACGCCGCGAGGGCGAAG } 60.0 \\
\text { GGGCGTTACAGGCGGAACGCGCTGCGGGAGGACGCCGCGAGGGCGAAG }\end{array}$} \\
\hline
\end{tabular}

mutations were also identified, namely, a G-insertion that we designated as $W u O$ siaa $23+1(\mathrm{~g})$ and a T-insertion we called WuOsiaa23+1(t) (Fig. 2b). In theory, the $\mathrm{KaO}$ siaa23-3 mutant gene encodes a protein that lacks an alanine (Ala, A), whereas the KaOsiaa23-39 protein lacks 13 amino acids (Fig. 2c). However, all the mutations did not affect the core sequence GWPPV of Domain II. For the Wuyunjing24 mutants, both types of single-base insertions are predicted to cause a frame shift in the OSIAA23 gene, thereby resulting in the deletion of Domains III and IV.

To assess the prevalence of off-target mutations and to further confirm the focal sequence changes, the mutants were re-sequenced and screened for mutation in a genome-wide scale. Firstly, all the genes in the IAA gene family and ARF gene family were investigated to check their mutations in the mutants. Except OsIAA23, all the other genes in the Kasalath and Wuyunjing 24 mutant genomes showed the same genotype with the corresponding wild-type genome (Supplementary Material 3 ). We then surveyed the genome-wide variant sites in each mutant in each background. In the three Kasalath mutants, 116 SNPs were called, with 106 in the intergenic region, 5 in the intron region, 3 in the UTR region and 2 in the exon region (Supplementary Figure S1A and Supplementary Material 3). The 2 SNPs located in the two different gene loci, Os02g0618200 and Os $10 \mathrm{~g} 0147400$, resulting a synonymous mutation and a single amino acid change in the non-domain region, respectively. Except the intended deletions due to the role of CRISPR/Cas9, only 4 INDELs were called in the Kasalath mutants, including 3 in the intergenic region and 1 in the intron region. In the two Wuyunjing 24 mutants, $W u O$ siaa $23+1(\mathrm{~g})$ and $\mathrm{WuO}$ siaa $23+1(t), 61$ SNPs and 12 INDELs were totally identified, except the G/T-insertion (Supplementary Figure S1B and Supplementary Material 3). Only 2 SNPs located in the exon region while all the other SNPs and INDELs were found in the non-coding regions. The 2 SNPs also resided in two different gene loci, Os06g0129000 and Os $11 \mathrm{~g} 0670700$, both encoding hypothetical proteins, without function reports.

\section{Phenotypic assessment of Osiaa23 mutant}

The T1 generation seeds of the heterozygous mutants of Kasalath and Wuyunjing24 were grown in Murashige and Skoog (MS) basal medium for 1 days under the same conditions for observation and statistical analysis. For Kasalath, of the 20 mutant plants, 10 were heterozygous

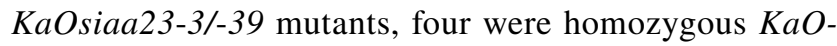
siaa23-3 mutants, and the remaining six were homozygous KaOsiaa23-39 mutants (Supplementary Table S4). For Wuyunjing24, of the 30 mutant plants, 15 were heterozygous $W u O$ siaa $23+1(g) /+1(t)$ mutants, eight were homozygous $W u O$ siaa $33+1(\mathrm{~g})$ mutants, and the remaining seven were homozygous $\mathrm{WuOsiaa23}+1(\mathrm{t})$ mutants (Supplementary Table S4).

In general, all the mutants in the seedling stage showed similar mutant phenotypes in height, crown root, primary root, and lateral root (Fig. 3). First, we found that all the mutants were of smaller stature than the two wild-types (Figs. 3a, b, 4a and Supplementary Table S4). Among these, mutants KaOsiaa23-3/-39 and KaOsiaa23-39 exhibited severe dwarfism $(\leq 10 \mathrm{~cm})$, significantly shorter than Kasalath. Second, except for mutant KaOsiaa23-3, all the other mutants produced significantly fewer crown roots. Meanwhile, the length of the crown roots of all the mutants was significantly shorter than for the two wild-types (Figs. 3a-d, $4 \mathrm{~b}$ and Supplementary Table S4). Also known as adventitious roots, crown roots are the main roots of rice plants, and a reduction in their number and length reduces seedling height. Third, mutants KaOsiaa-3/-39 and KaOsiaa-39 showed extreme inhibition of lateral root formation. Another Kasalath mutant, KaOsiaa-3, showed slightly fewer lateral roots than the wild-type Kasalath. Similarly, all the Wuyunjing24 mutants also showed slight inhibition of lateral root elongation, consistence with the reduced number of crown roots (Figs. 3a-d, $4 \mathrm{c}$ and Supplementary Table S4). This agrees with the previously reported phenotype of Osiaa23 homozygous mutants (Ni et al. 2011). Lateral roots play an important role in rice; these are responsible for nutrient and water uptake. The inhibition of lateral root formation and elongation might be responsible for the observed abnormal 
Fig. 3 Phenotypes of 7-day-old seedlings of wild type plants

(WT), mutants of Osiaa23. Phenotype of 7-day-old seedlings of wild-type and mutants of Kasalath (a) and Wuyunjing24 (b), $\mathrm{Bar}=2.5 \mathrm{~cm}$. As marked, from left to right: wild-type (WT), the heterozygous mutants, and the homozygous mutants. Root phenotype of 7-day-old seedlings of wild-type and mutants of Kasalath (c) and Wuyunjing24 (d), Diameter $=1.4 \mathrm{~cm}$. Enlarge views of root of the heterozygous mutants of Kasalath (e) and Wuyunjing24 (f)

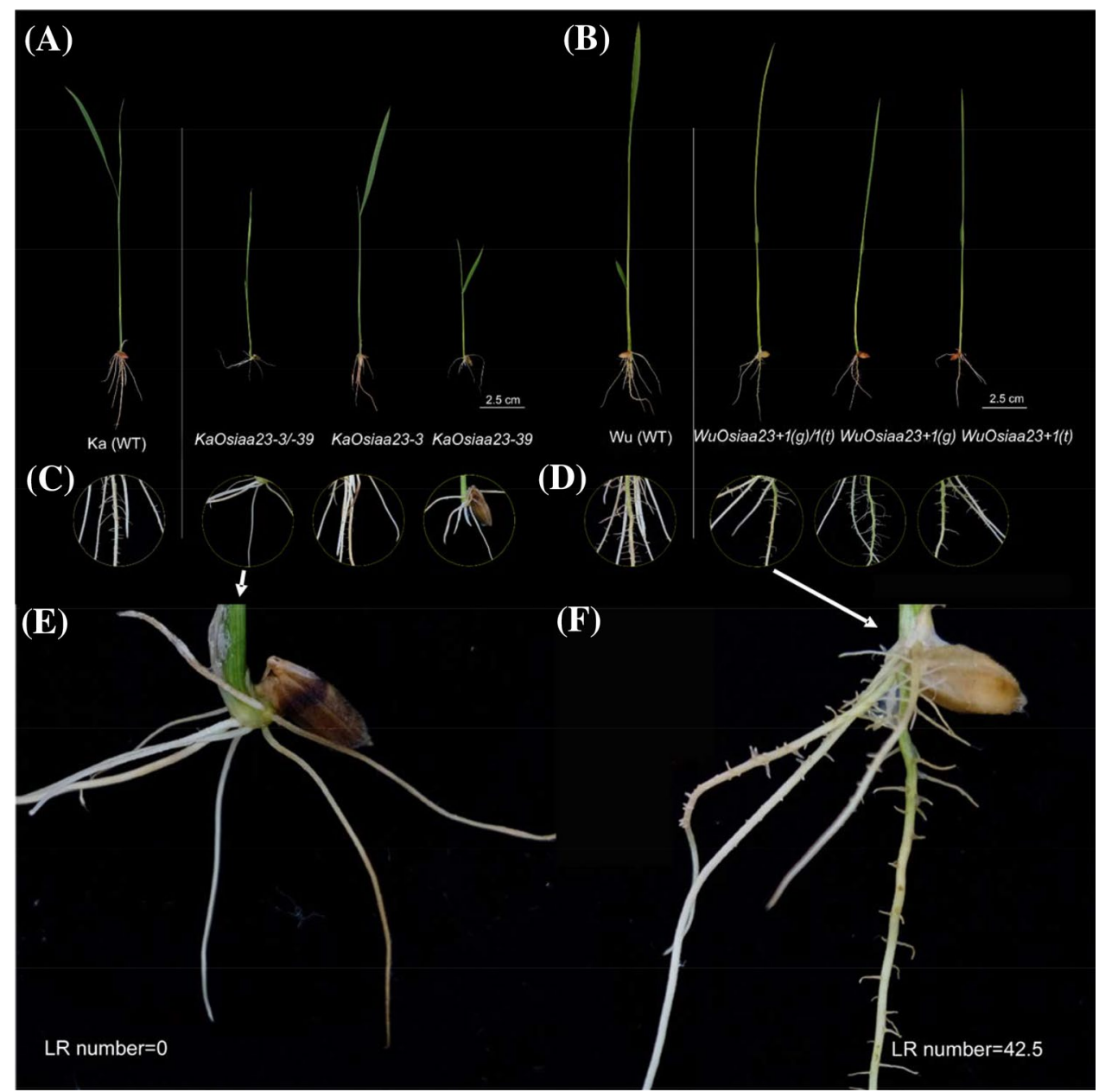

mutant phenotype. Finally, the primary root length was similar to that of the shoot and crown root lengths, although no significant reduction was detected in the mutant $\mathrm{KaO}$ siaa-3. Similarly, the fresh weight of the total roots of all six mutants was significantly lower than for the two wild-types (Fig. 4d-f and Supplementary Table S4).

Seven-day-old seedlings were transplanted into planting boxes and placed outdoors. Wild-type plants and mutants of Kasalath and Wuyunjing24 were planted in the same box. After about 125 days and 145 days, all the wild-type plants and mutants of Kasalath and Wuyunjing24 reached the full ripening stage, respectively (Supplementary Table S5). Compared to the wild-type, the number of tillers of the two mutants, KaOsiaa23-3/-39 and KaOsiaa23-39, was significantly reduced, whereas the other mutants showed no special phenotype in tiller number (Fig. 5 and Supplementary Table S5). However, the height of all mature plant mutants was significantly lower than that of the two wild-types (Fig. 5a, b and Supplementary Table S5). Especially for the two mutants carrying the 39-bp deletion, these were about $30 \mathrm{~cm}$ shorter than the wild Kasalath. Another important and distinct mutant phenotype is their seed setting rate. All the mutants had a significantly lower seed setting rate compared to the wild-type, indicating that the OSIAA23 gene indirectly affects rice production. However, the production of the mutants also significantly varied. The mutant $\mathrm{KaO}$ siaa23-3 had the same ear type as the wild Kasalath, and all the seeds are plump and full seeds (Fig. 5c). In contrast, the spikelets of the two mutants carrying the 39-bp deletion had no pedicels and the stalk supporting the spikelet on the panicle branch, and most seeds were empty and blighted, thereby resulting in an extremely low seed setting rate.

\section{Quantification of OsIAA23 mRNA expression}

The OsIAA23 gene had the similar expression profile in the wild-type Kasalath and Wuyunjing24, exhibiting the highest expression levels in the roots, followed by the stem, and showed less expression in the other tissues such as the sheaths, leaves, flowers, and seeds (Fig. 6a). This expression pattern coincides with the phenotype of the Osiaa23 mutants that we observed, which had defects involving initiation of crown roots and lateral roots, and with respect to overall height. 
Fig. 4 Growth parameters of wild type plants (WT), mutants of Osiaa23. The phenotypes of Osiaa 23 mutants are compared with WT in the aspects of shoot length (a), crown root number (b), lateral root number (c), primary root length $(\mathbf{d})$, crown root length (e), total root (fresh) weight (f), tiller number (g), mature plant height (h) and seed setting rate (i). Black columns represent Kasalath mutants, and gray columns represent Wuyunjing24 mutants. As marked at the bottom, from left to right: wild-type plants (WT), the heterozygous mutants, and the homozygous mutants. The standard deviation of the mean was denoted as error bar for each column. $* p<0.05$, $* * p<0.01$ (Student's $t$ test)
(A)

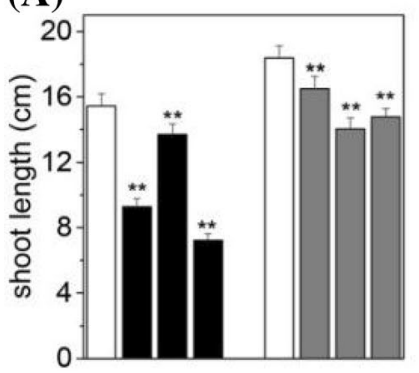

(D)

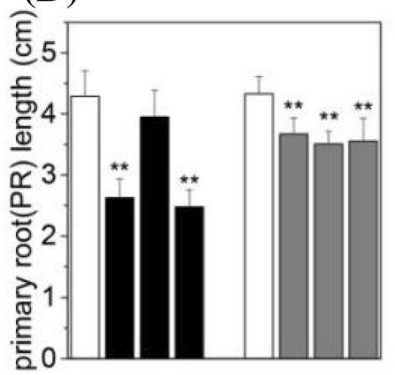

(G)

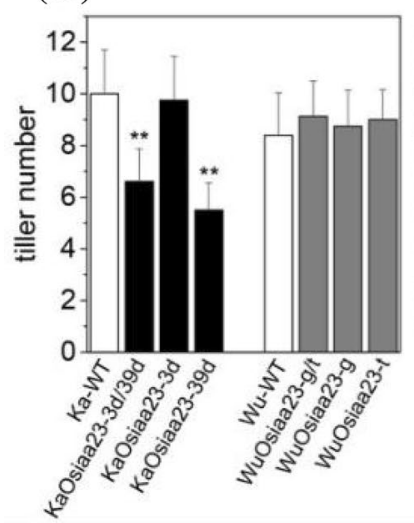

(B)

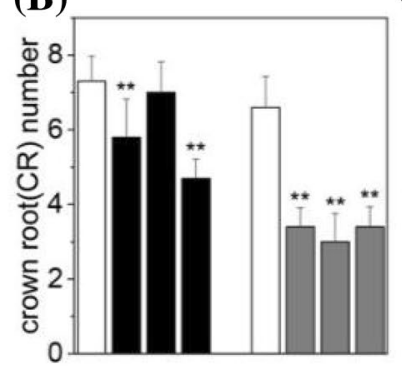

(E)

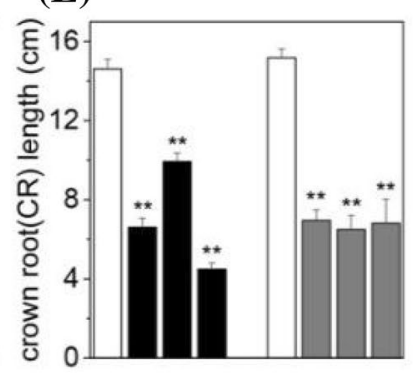

(H)

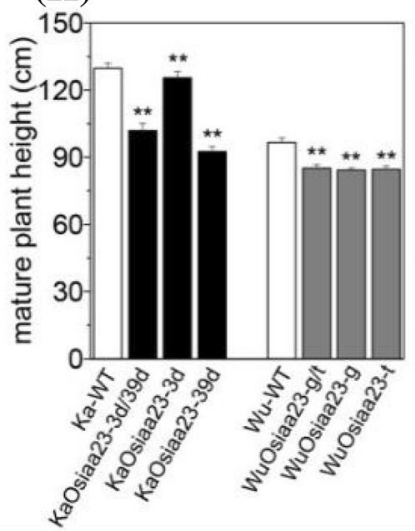

(C)

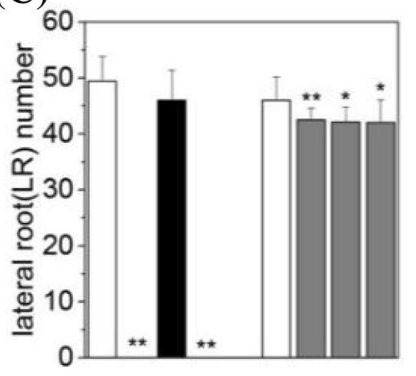

(F)

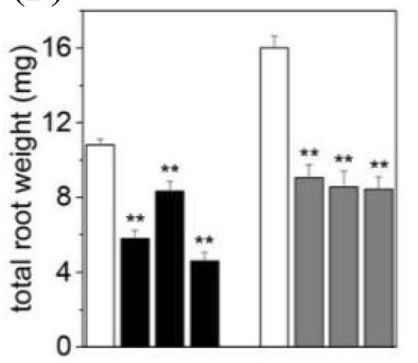

(I)

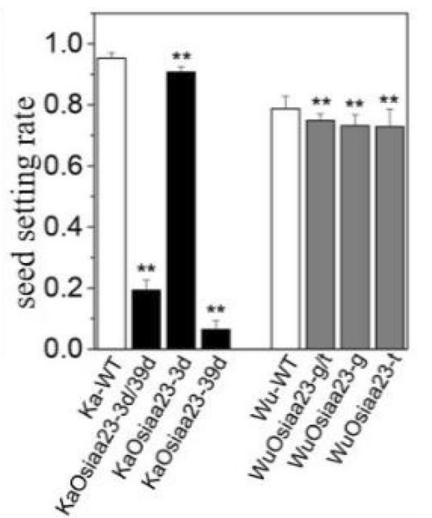

To test whether the two homologs of OsIAA23, OsIAA1 and OsIAA15, could compensate the function of destroyed Osiaa23 in the mutants, RT-PCR analysis of the roots of the mutants and its wild-type plants was performed to assess their expression levels (Fig. 6b). The three homologs exhibited similar expression profiles between wild-type Kasalath and Wuyunjing24, i.e., OSIAA23 and OSIAA15 had a higher expression levels, whereas OsIAAl had a relatively lower expression level. For the Kasalath mutants, all showed a reduction in expression of edited OSIAA23, particularly the homozygous mutants $\mathrm{KaOsiaa23-39}$. It seems that lower Osiaa23 expression leads to a more striking mutant phenotype in the Kasalath variety. Interestingly, the other two homologs, particularly OSIAA15, show a similar downregulated expression pattern as that of Osiaa23, suggesting that the two homologs do not compensate for the function of Osiaa23, but that the three genes seem to cooperate together in a co-expression pattern. The expression of
Osiaa 23 was slightly decreased in the homolog mutant and further decreased in the heterozygous mutant, whereas the expression of OSIAAI and OSIAAI5 was not reduced in the mutants. The mutation type in Kasalath is a three-nucleotide deletion, whereas a single base-pair insertion, which may result in frameshift, was found in Wuyunjing24. Then, we expect that the phenotypic change in Wuyunjing24 mutants should be more obvious and significant. Meanwhile, the transcripts of the Wuyunjing24 Osiaa23 mutant might also be degraded because of premature transcription termination. However, a slightly mutant phenotype and high expression levels of the mutated Osiaa23 were observed in the Wuyunjing24 mutants. Is it possible that the mutated Osiaa 23 gene in Wuyunjing24 underwent an alteration in transcription to avoid premature termination?

To assess this question, we examined the genome file of O. sativa (IRGSP 1.0), which contains two annotated transcripts (Os06t0597000-01 and Os06t0597000-02), encoding 


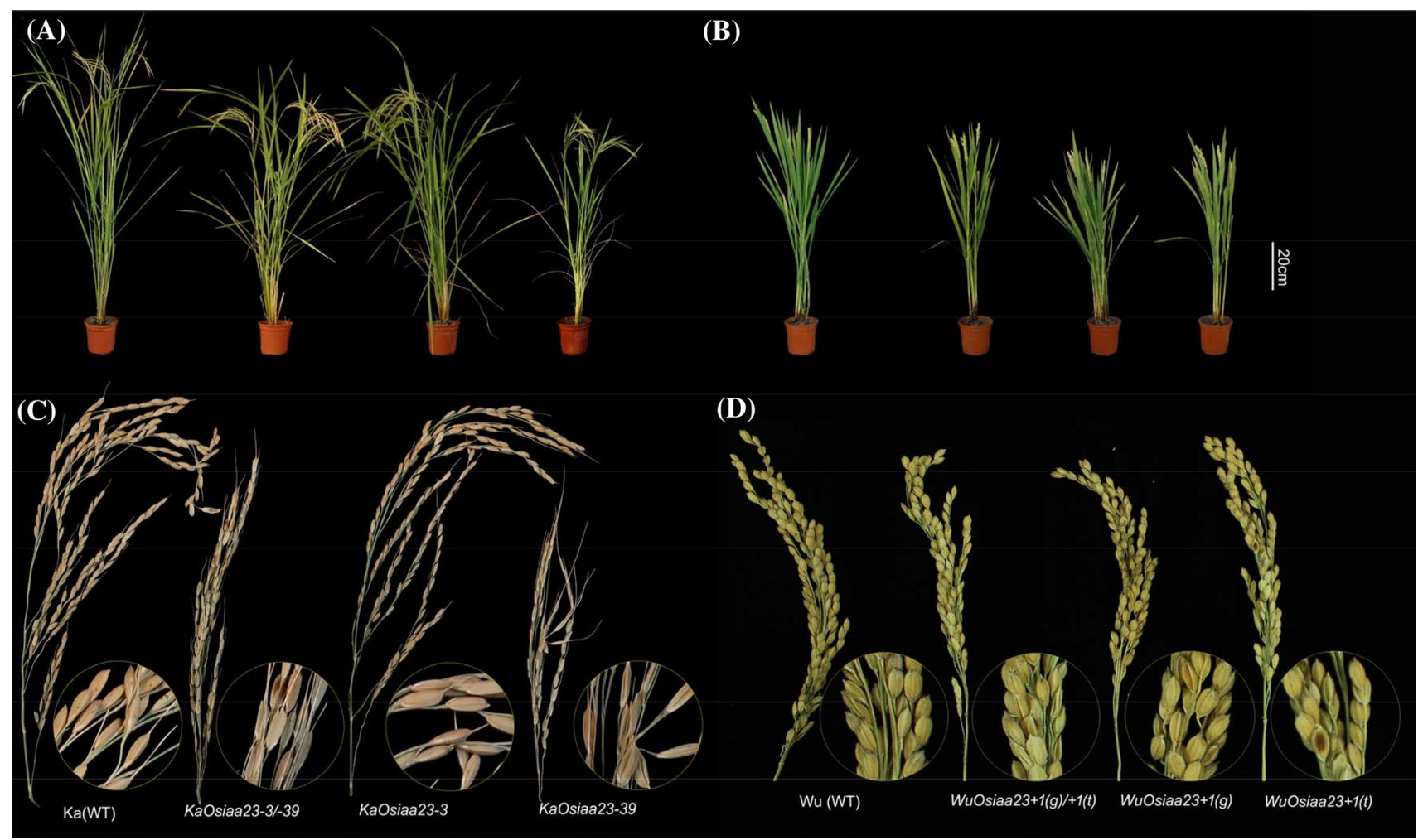

Fig. 5 Phenotypes of mature plants of wild type (WT) and mutants of Osiaa23. Phenotype of plants at full ripening stage of wild-type (WT) and mutants of Kasalath (a) and Wuyunjing24 (b), Bar=20 cm. Phe-

proteins of 193 and 262 amino acids, respectively. Only the last 27 amino acids are identical in the two predicted proteins. We interrogated public RNA-Seq data of rice to determine whether the OsIAA23 gene has other transcripts. Interestingly, we found several new exon-intron junctions (Supplementary Figure S1). Two of the new junctions are respectively 11-bp (chr06:23502684-23502763) and 13-bp (chr06:23502686-23502763) distal to the annotated second intron of the transcript Os06t0597000-01. If a single base-pair insertion was induced in the second exon before the second intron, just as we found in the edited Osiaa 23 in Wuyunjing 24 mutants, then the gene could adopt a new transcription pattern with the new junction producing a functional mRNA. For Os06t0597000-01, the new predicted mRNA would translate a novel mosaic protein, with the former 71 amino acids similar to the protein translated by Os06t0597000-01 and the latter 127 amino acids the same with the protein translated by Os06t0597000-02 (Fig. 6c). For Os06t0597000-02, on the other hand, the predicted mRNA would translate a new auxin/IAA protein, as indicated in Fig. 6c. By analyzing RNA-seq produced by Illumina Hiseq 4000 in wild-type Wuyunjing 24 and mutant plants, we were indeed able to detect these two new junctions (chr06:23502684-23502763 and chr06:23502686-23502763) notypes of spikelet of wild-type (WT), mutants of Kasalath (c) and Wuyunjing24 (d). As marked at the bottom, from left to right: wildtype (WT), the heterozygous mutants, and the homozygous mutants

(Supplementary Table S6). Although the vast majority of the exon-intron junctions in the wild type and mutants are still the annotated ones (99\%), the proportion of the two new junctions in WuOsiaa23 mutants increased by 2 to ninefold relative to the wild Wuyunjing 24 control. Similarly, the junction chr06:23502686-23502763 was also found in Kasalath and KaOsiaa23 mutants while the junction chr06:23502684-23502763 was only observed in the wild Kasalath control (Supplementary Table S7). The Pacbio data of RNA-seq was successful in resolving the puzzle by providing the complete sequences of the new transcript isoforms predicted in Fig. 6c.

\section{Discussion}

\section{Pleiotropic effects of OsIAA23 gene on rice and its role in grass}

Traditionally, plant mutants have been generated by ethyl methanesulfonate (EMS), irradiation, T-DNAs or transposons. These mutagenetic methods are usually random and often labor-intensive and time-consuming for identification the causal mutation. The CRISPR/Cas9 gene editing 


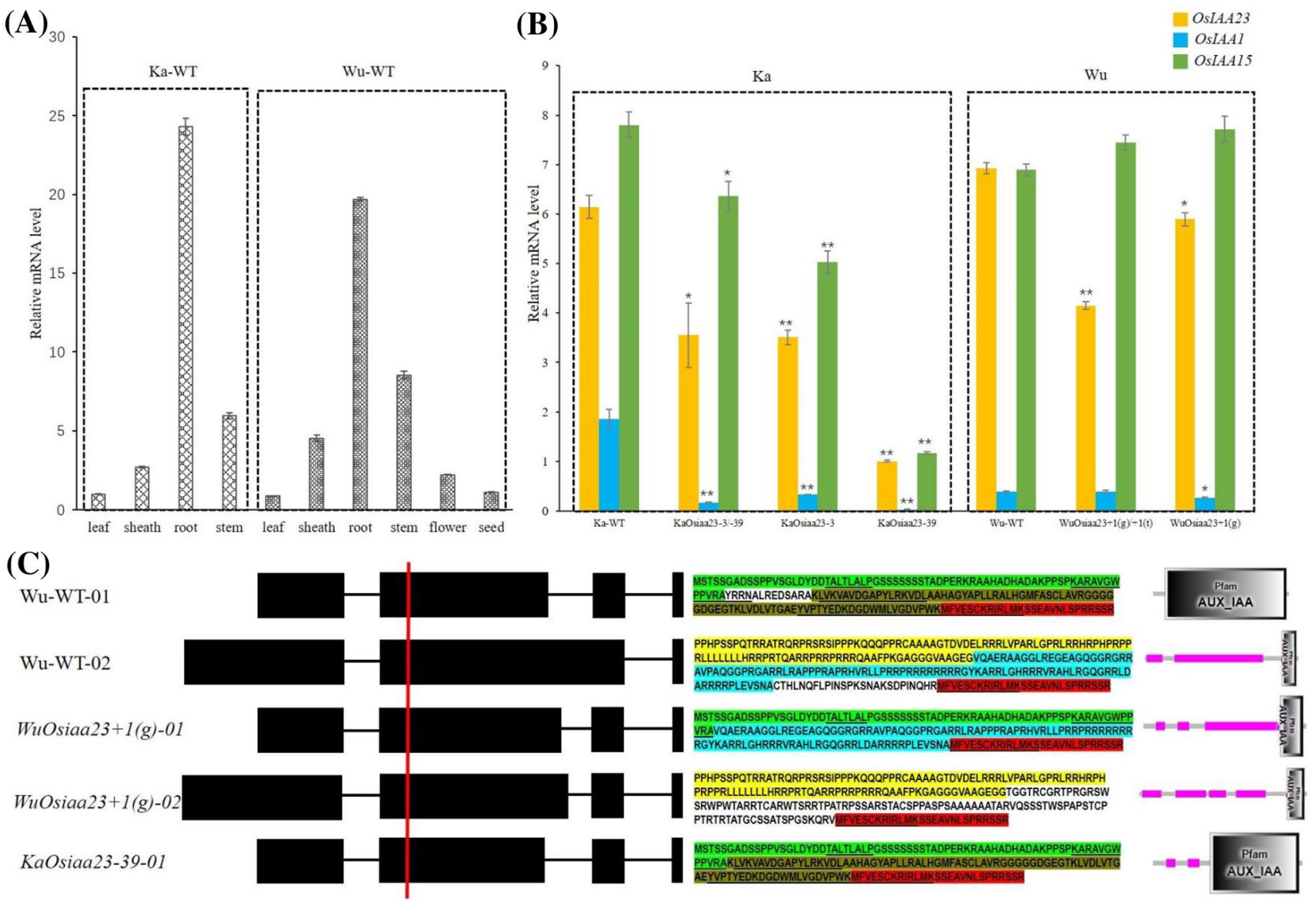

Fig. 6 Expression models of OsIAA23 and its homologs. Real-time PCR expression profiles of OsIAA23 gene in different tissues of wild-type (Ka-WT) and Wuyunjing24 (Wu-WT) (a). Comparison of expression levels of OSIAA23, OsIAA1 and OsIAA15 in the root of different mutants $(\mathbf{b})$. The predicted transcripts, protein sequences and structures of OsIAA23 gene and edited Osiaa 23 gene (c). The

technology could provide precise and highly efficient modifications in specific regions. It also allows rapid identification of causal mutations. Here, we used the CRISPR/Cas9 system and isolated several Osiaa23 mutants in japonica (Wuyunjing24) and indica (Kasalath) rice. Both heterozygous double knockout mutants were obtained, with deletions in Kasalath and insertions in Wuyunjing24, indicating the high efficiency and diversity of the editing function of the CRISPR/Cas9 system.

The previously reported EMS-mutagenized Osiaa23 mutant, which is generated by a point mutation in the core sequence of Domain II, exhibits pleiotropic defects in its root tissues, including crown roots, lateral roots, and root cap (Ni et al. 2011). In the former study, the heterozygous mutants were intermediate in phenotype and exhibited normal fertility, whereas the homozygous mutant showed loss of lateral and crown root primordia, the root gravitropic response and abnormal fertility. An amino acid substitution red vertical line marks the position of the gene editing. The amino acid sequences highlighted with the same color are the same. The sequences of Domain I, II, III and IV are indicated orderly by underline text. The standard deviation of the mean was denoted as error bar for each column. ${ }^{*} p<0.05,{ }^{* *} p<0.01$

( $\mathrm{G}$ to $\mathrm{E}$ ) in the conserved core sequence of Domain II of the OsIAA23 protein could cause pleiotropic defects in growth and development, suggesting the importance of the core sequence GWPPV. Here, we generated several mutants using mutations just following the core sequence GWPPV. In the Kasalath mutants, three-nucleotide and 39-nucleotides deletions were detected, resulting in an alanine deletion and a 13-amino acid deletion, respectively. Mutants $\mathrm{KaO}$ siaa23-3/-39 and KaOsiaa23-39, both carrying the 39-bp deletion, showed the most obvious and significant mutant phenotypes, especially the homozygous mutant. Defects in the root tissues of mutants then result in extremely low fertility rates. In contrast, the deletion of the alanine residue after the core sequence of the Domain II in mutant KaOsiaa23-3 mainly resulted in limited crown root length. This indicates that the absence of these amino acids affects the normal metabolism of the OsIAA23 protein, but the degree of reduction was weaker than the previously reported Osiaa23, 
suggesting the importance of the core sequence GWPPV of Domain II.

The root system of monocots and dicots differs in terms of architecture (Smith and De Smet 2012). Most dicots have a primary root and several lateral roots. The grasses often have a fibrous root system and the branching roots are produced through crown roots. Crown roots are much more vital for the root architecture of grasses than in dicots. Auxin induces lateral-root formation in dicots and crown root formation in grasses (McSteen 2010). It appears that crown root initiation is similarly controlled by auxin to initiate lateral root formation. However, crown roots are developed from shoots, whereas lateral roots are produced from root tissues. There thus might be specific genes that are regulated by auxin that control crown root growth in grasses. Here, no OsIAA23 homolog (amino acids similarity $>30 \%$ ) was identified in A. thaliana, and the OsIAA23 gene is specific to grass. One of the main functions of OSIAA23 is related to crown roots or adventitious roots, which is grass-specific. It is possible that the grass-specific OsIAA23 gene was generated after the appearance of the grasses for crown root initiation. About seven OsIAA genes in rice have been reported, only OsIAA23 was found to have the effect on the crown roots formation (Nakamura et al. 2006; Song et al. 2009; Ni et al. 2011, 2014; Zhu et al. 2012; Kitomi et al. 2012; Song and Xu 2013). Thus, the grass-specific OsIAA23 gene might play one of the major roles for root architecture in grasses.

\section{Interactions among OsIAA23 and its two homologs}

Aux/IAA proteins are usually characterized by four highly conserved domains I-IV (Mukesh et al. 2006). Domain I functions as an active repression domain, and Domain II is responsible for the protein Aux/IAA stability. Some Aux/ IAA proteins lack an apparent Domain II, and some lack both Domains I and II and do not function as repressors in the auxin signaling pathway (Tiwari et al. 2004; Dreher et al. 2006). Domains III and IV are protein-protein interaction domains, facilitating the formation of ARF-ARF, ARFAux/IAA and Aux/IAA- Aux/IAA homo- and heterodimers. As auxin response factors, Aux/IAA proteins usually form multimers with ARFs. Recently, many studies on interactions between Aux/IAA and Aux/IAA proteins have been reported. Based on large-scale interactome analyses, such as yeast two-hybrid, bimolecular fluorescence complementation (BiFC) experiments, a comprehensive physical interactome map of Aux/IAA proteins has been developed in Arabidopsis thaliana, indicating Aux/IAA-Aux/IAA interactions are common (Vernoux et al. 2011; Luo et al. 2018). In maize, ZmIAA15 was found to have interaction both with
ZmIAA10 and ZmIAA29. And ZmIAA23 also interacted with ZmIAA10 as well as ZmIAA29 (Ludwig et al. 2014).

Here, only Domain II was damaged in Osiaa23 in Kasalath mutants, and Domains I, III, and IV remained intact. The expression of Osiaa 23 sharply decreased, particularly in the homozygote mutant KaOsiaa23-39, which harbored a 13-amino acid deletion downstream of core of Domain II. With the decrease in the expression level of Osiaa23, its two homologs, OsIAAI and OSIAA15, also showed reduced expression. It seems that in the Kasalath mutants, Osiaa23 and its two homologs cooperate or are involved in the same pathway such as auxin signaling. The three proteins might form hetero-oligomers as they all have the intact interaction domains III and IV. Their interactions result in the observed mutant phenotype. In Wuyunjing24 mutants, although the expression level of Osiaa23 slightly decreased, the expression of OsIAAI and OSIAA15 did not significantly decrease. This might be because the edited Osiaa 23 has recovered its partial function, and its partners function normally. Unlike the Kasalath mutants, the Wuyunjing 24 mutants harbor a frameshift mutation in Osiaa23, which might be eliminated by alternative splicing, resulting in a weak mutant phenotype. Based on the RT-PCR results, we predict that OSIAA23 might cooperate with its homologs, particularly OSIAA15. When the function of OSIAA23 is destroyed, the two homologs also reduce their expression; when the function of OsIAA23 is recovered, the two homologs retain normal expression level. More experiments are needed to test their relationships. It is possible that homodimers or heterodimers of Aux/IAA proteins might be involved with the regulation of auxin response genes. Then the Aux/IAA homo- or hetero-dimers form higher order multimers with ARF proteins and block the transcriptional activation of auxin-responsive genes.

Acknowledgements This work was supported by supported by the National Natural Science Foundation of China (31570368, 31870204), the National Major Special Project on New Varieties Cultivation for Transgenic Organisms (No. 2016ZX08009001-003) and Jiangsu Collaborative Innovation Center for Modern Crop Production.

Author contributions $\mathrm{XZ}$ and SY conceived and designed the experiments; MJ, HH and JK performed the experiments; MJ, XZ and SY analyzed the data and wrote the paper. M.B.T edited the paper and participated in the discussion.

\section{Compliance with ethical standards}

Conflict of interest Authors do not have any conflicts of interest.

Open Access This article is distributed under the terms of the Creative Commons Attribution 4.0 International License (http://creativecommons.org/licenses/by/4.0/), which permits unrestricted use, distribution, and reproduction in any medium, provided you give appropriate credit to the original author(s) and the source, provide a link to the Creative Commons license, and indicate if changes were made. 


\section{References}

Çakir B, Kiliçkaya O, Olcay AC (2012) Genome-wide analysis of Aux/IAA genes in Vitis vinifera: cloning and expression profiling of a grape Aux/IAA gene in response to phytohormone and abiotic stresses. Acta Physiol Plant 35:365-377

DePristo MA, Banks E, Poplin R, Garimella KV, Maguire JR, Hartl C, Philippakis AA, del Angel G, Rivas MA, Hanna M, McKenna A, Fennell TJ, Kernytsky AM, Sivachenko AY, Cibulskis K, Gabriel SB, Altshuler D, Daly MJ (2011) A framework for variation discovery and genotyping using next-generation DNA sequencing data. Nat Genet 43:491-498. https://doi. org/10.1038/ng.806

Dreher KA, Brown J, Saw RE, Callis J (2006) The Arabidopsis Aux/ IAA protein family has diversified in degradation and auxin responsiveness. Plant Cell 18:699-714. https://doi.org/10.1105/ tpc. 105.039172

Fukaki H, Tameda S, Masuda H, Tasaka M (2002) Lateral root formation is blocked by a gain-of-function mutation in the SOLITARY-ROOT/IAA14 gene of Arabidopsis. Plant J Cell Mol Biol 29:153-168

Fukaki H, Nakao Y, Okushima Y, Theologis A, Tasaka M (2005) Tissue-specific expression of stabilized SOLITARY-ROOT/ IAA14 alters lateral root development in Arabidopsis. Plant J Cell Mol Biol 44:382-395. https://doi.org/10.1111/j.1365313X.2005.02537.x

Gao J, Cao X, Shi S, Ma Y, Wang K, Liu S, Chen D, Chen Q, Ma H (2016) Genome-wide survey of Aux/IAA gene family members in potato (Solanum tuberosum): identification, expression analysis, and evaluation of their roles in tuber development. Biochem Biophys Res Commun 471:320-327. https://doi.org/10.1016/j. bbrc.2016.02.013

Jung H, Lee D-K, Choi YD, Kim J-K (2015) OsIAA6, a member of the rice Aux/IAA gene family, is involved in drought tolerance and tiller outgrowth. Plant Sci 236:304-312. https://doi. org/10.1016/j.plantsci.2015.04.018

Kepinski S, Leyser O (2004) Auxin-induced SCFTIR1-Aux/IAA interaction involves stable modification of the SCFTIR1 complex. Proc Natl Acad Sci USA 101:12381-12386. https://doi. org/10.1073/pnas.0402868101

Kitomi Y, Inahashi H, Takehisa H, Sato Y, Inukai Y (2012) OsIAA13-mediated auxin signaling is involved in lateral root initiation in rice. Plant Sci 190:116-122. https://doi. org/10.1016/j.plantsci.2012.04.005

Kumar S, Stecher G, Tamura K (2016) MEGA7: molecular evolutionary genetics analysis version 7.0 for bigger datasets. Mol Biol Evol 33:1870-1874. https://doi.org/10.1093/molbev/ msw054

Li H (2013) Aligning sequence reads, clone sequences and assembly contigs with BWA-MEM. arxiv:13033997 Q-Bio

Li H, Wang B, Zhang Q, Wang J, King GJ, Liu K (2017) Genome-wide analysis of the auxin/indoleacetic acid (Aux/IAA) gene family in allotetraploid rapeseed (Brassica napus L.). BMC Plant Biol 17:204. https://doi.org/10.1186/s12870-017-1165-5

Liscum E, Reed JW (2002) Genetics of Aux/IAA and ARF action in plant growth and development. Plant Mol Biol 49:387-400

Ludwig Y, Zhang Y, Hochholdinger F (2013) The maize (Zea mays L.) AUXIN/INDOLE-3-ACETIC ACID gene family: phylogeny, synteny, and unique root-type and tissue-specific expression patterns during development. PLoS ONE 8:e78859. https://doi. org/10.1371/journal.pone.0078859

Ludwig Y, Berendzen KW, Xu C, Piepho H-P, Hochholdinger F (2014) Diversity of stability, localization, interaction and control of downstream gene activity in the maize Aux/IAA protein family. PLoS ONE 9:e107346. https://doi.org/10.1371/journ al.pone. 0107346

Luo S, Li Q, Liu S, Pinas NM, Tian H, Wang S (2015) Constitutive expression of OsIAA9 affects starch granules accumulation and root gravitropic response in Arabidopsis. Front Plant Sci 6:1156. https://doi.org/10.3389/fpls.2015.01156

Luo J, Zhou J-J, Zhang J-Z (2018) Aux/IAA gene family in plants: molecular structure, regulation, and function. Int J Mol Sci 19:259. https://doi.org/10.3390/ijms19010259

Mai Y-X, Wang L, Yang H-Q (2011) A gain-of-function mutation in IAA7/AXR2 confers late flowering under short-day light in Arabidopsis. J Integr Plant Biol 53:480-492. https://doi.org/10 $.1111 / \mathrm{j} .1744-7909.2011 .01050 . \mathrm{x}$

McSteen P (2010) Auxin and monocot development. Cold Spring Harb Perspect Biol 2:a001479. https://doi.org/10.1101/cshpe rspect.a001479

Miao J, Guo D, Zhang J, Huang Q, Qin G, Zhang X, Wan J, Gu H, Qu L-J (2013) Targeted mutagenesis in rice using CRISPRCas system. Cell Res 23:1233-1236. https://doi.org/10.1038/ cr. 2013.123

Mukesh J, Navneet K, Rohini G, Jitendra KT, Akhilesh KT, Khurana JP (2006) Structure and expression analysis of early auxin-responsive Aux/IAA gene family in rice (Oryza sativa). Funct Integr Genomics 6:47-59. https://doi.org/10.1007/s10142-005-0005-0

Nakamura A, Umemura I, Gomi K, Hasegawa Y, Kitano H, Sazuka T, Matsuoka M (2006) OsIAA3-Production and characterization of auxin-insensitive rice by overexpression of a mutagenized rice IAA protein. Plant J 46:297-306. https://doi.org/10.1111/j.1365313X.2006.02693.X

Ni J, Wang G, Zhu Z, Zhang H, Wu Y, Wu P (2011) OsIAA23mediated auxin signaling defines postembryonic maintenance of QC in rice. Plant J 68:433-442. https://doi.org/10.1111/j.1365313X.2011.04698.x

Ni J, Zhu Z, Wang G, Shen Y, Zhang Y, Wu P (2014) Intragenic suppressor of Osiaa23 revealed a conserved tryptophan residue crucial for protein-protein interactions. PLoS ONE 9:e85358. https ://doi.org/10.1371/journal.pone.0085358

Ouellet F, Overvoorde PJ, Theologis A (2001) IAA17/AXR3: biochemical insight into an auxin mutant phenotype. Plant Cell 13:829-841. https://doi.org/10.1105/tpc.13.4.829

Overvoorde PJ, Okushima Y, Alonso JM, Chan A, Chang C, Ecker JR, Hughes B, Liu A, Onodera C, Quach H, Smith A, Yu G, Theologis A (2005) Functional genomic analysis of the AUXIN/INDOLE3-ACETIC ACID gene family members in Arabidopsis thaliana. Plant Cell 17:3282-3300. https://doi.org/10.1105/tpc.105.036723

Reed JW (2001) Roles and activities of Aux/IAA proteins in Arabidopsis. Trends Plant Sci 6:420-425. https://doi.org/10.1016/S1360 $-1385(01) 02042-8$

Rogg LE, Lasswell J, Bartel B (2001) A gain-of-function mutation in IAA28 suppresses lateral root development. Plant Cell 13:465-480

Singh VK, Jain M (2015) Genome-wide survey and comprehensive expression profiling of Aux/IAA gene family in chickpea and soybean. Front Plant Sci 6:918. https://doi.org/10.3389/ fpls.2015.00918

Smith S, De Smet I (2012) Root system architecture: insights from Arabidopsis and cereal crops. Philos Trans R Soc B Biol Sci 367:1441-1452. https://doi.org/10.1098/rstb.2011.0234

Song Y, Xu Z-F (2013) Ectopic overexpression of an AUXIN/ INDOLE-3-ACETIC ACID (Aux/IAA) Gene OsIAA4 in rice induces morphological changes and reduces responsiveness to auxin. Int J Mol Sci 14:13645-13656. https://doi.org/10.3390/ ijms 140713645

Song Y, You J, Xiong L (2009) Characterization of OsIAA1 gene, a member of rice Aux/IAA family involved in auxin and 
brassinosteroid hormone responses and plant morphogenesis. Plant Mol Biol 70:297-309. https://doi.org/10.1007/s1110 3-009-9474-1

Tatematsu K, Kumagai S, Muto H, Sato A, Watahiki MK, Harper RM, Liscum E, Yamamoto KT (2004) MASSUGU2 encodes Aux/IAA19, an auxin-regulated protein that functions together with the transcriptional activator NPH4/ARF7 to regulate differential growth responses of hypocotyl and formation of lateral roots in Arabidopsis thaliana. Plant Cell 16:379-393. https://doi. org/10.1105/tpc.018630

Teale WD, Paponov IA, Palme K (2006) Auxin in action: signalling, transport and the control of plant growth and development. Nat Rev Mol Cell Biol 7:847-859. https://doi.org/10.1038/nrm2020

Thorvaldsdóttir H, Robinson JT, Mesirov JP (2013) Integrative genomics viewer (IGV): high-performance genomics data visualization and exploration. Brief Bioinform 14:178-192. https://doi. org/10.1093/bib/bbs017

Tian Q, Uhlir NJ, Reed JW (2002) Arabidopsis SHY2/IAA3 inhibits auxin-regulated gene expression. Plant Cell 14:301-319

Tiwari SB, Hagen G, Guilfoyle TJ (2004) Aux/IAA proteins contain a potent transcriptional repression domain. Plant Cell 16:533-543. https://doi.org/10.1105/tpc.017384

Vernoux T, Brunoud G, Farcot E, Morin V, den Daele HV, Legrand J, Oliva M, Das P, Larrieu A, Wells D, Guédon Y, Armitage L, Picard F, Guyomarc'h S, Cellier C, Parry G, Koumproglou R, Doonan JH, Estelle M, Godin C, Kepinski S, Bennett M, Veylder LD, Traas J (2011) The auxin signalling network translates dynamic input into robust patterning at the shoot apex. Mol Syst Biol 7:508. https://doi.org/10.1038/msb.2011.39

Woodward AW, Bartel B (2005) Auxin: regulation, action, and interaction. Ann Bot 95:707-735. https://doi.org/10.1093/aob/mci083
Worley CK, Zenser N, Ramos J, Rouse D, Leyser O, Theologis A, Callis J (2000) Degradation of Aux/IAA proteins is essential for normal auxin signalling. Plant J 21:553-562. https://doi. org/10.1046/j.1365-313x.2000.00703.x

Yuan Y, Zhang Q, Zeng S, Gu L, Si W, Zhang X, Tian D, Yang S, Wang L (2017) Selective sweep with significant positive selection serves as the driving force for the differentiation of japonica and indica rice cultivars. BMC Genomics 18:307. https://doi. org/10.1186/s12864-017-3702-X

Zhang X, Yang S, Wang J, Jia Y, Huang J, Tan S, Zhong Y, Wang L, Gu L, Chen J-Q, Pan Q, Bergelson J, Tian D (2015) A genome-wide survey reveals abundant rice blast $\mathrm{R}$ genes in resistant cultivars. Plant J Cell Mol Biol 84:20-28. https://doi.org/10.1111/tpj.12955

Zhang Z, Li J, Tang Z, Sun X, Zhang H, Yu J, Yao G, Li G, Guo H, Li J, Wu H, Huang H, Xu Y, Yin Z, Qi Y, Huang R, Yang W, Li Z (2018) Gnp4/LAX2, a RAWUL protein, interferes with the OsIAA3-OsARF25 interaction to regulate grain length via the auxin signaling pathway in rice. J Exp Bot 69:4723-4737. https ://doi.org/10.1093/jxb/ery256

Zhu Z-X, Liu Y, Liu S-J, Mao C-Z, Wu Y-R, Wu P (2012) A gain-offunction mutation in OsIAA11 affects lateral root development in rice. Mol Plant 5:154-161. https://doi.org/10.1093/mp/ssr074

Publisher's Note Springer Nature remains neutral with regard to jurisdictional claims in published maps and institutional affiliations. 